\title{
Online Hotel Parcel and Payment System Using GPS and Android
}

\author{
Aniket Sahani ${ }^{1}$, Shivram Suravase ${ }^{2,}$ Chaitanya Ghule ${ }^{3}$, Prafulla Gavade ${ }^{4}$ \\ ${ }^{I}$ (Computer, Smt. Kashibai Navale College of Engineering/University of Pune, India) \\ ${ }^{2}$ (Computer, Smt. Kashibai Navale College of Engineering/ University of Pune, India) \\ ${ }^{3}$ (Computer, Smt. Kashibai Navale College of Engineering/ University of Pune, India) \\ ${ }^{4}$ (Computer, Smt. Kashibai Navale College of Engineering/ University of Pune, India)
}

\begin{abstract}
With the advent of GPS technologies more and more applications are getting developed on various independent platforms relating to services offered by smart devices such as smartphones, tablets, Pc's based on locations fetched by the GPS (Global positioning system). And with the world going online for purchasing their day to day basic things the need of clubbing GPS based services with other important sectors of the economy such as retail, hotel industry is the need of the fast becoming world.We would like to use location based services to help user to find good restaurants from its current place. Here we would like to propose location based food ordering and parcel system which will help user to place order from its location and save his/her time by making him the facility of paying his incurred amount online. Ones the user places his/her order, the application on the client side forwards the order to the server for checking the if the order is viable or not. After the order is verified by the server, a message is sent to the restaurant administrator consisting of the order and the delivery address of the user. Once the order is ready, the order is dispatched to the user/client with the process getting completed on receiving the delivery of the ordered food. Application also supports feedback and reviews which will be helpful to restaurants to improve their food and services quality.
\end{abstract}

Keywords : IP (Internet Protocol), JDK (Java Development Kit), KVM (Keyboard Video Mouse), LBS (Location Based Services), SDK (Software Development Kit).

\section{Introduction}

Location based service (LBS) is fast becoming a need of the smart devices getting developed based on Global Positioning System (GPS). With the users also getting smart enough, these applications are being used on daily basis by users worldwide for doing many things such as commuting from one place to another using applications like Google Maps, getting useful information about a place using the feeds provided by the people who have visited that place before, booking of hotels for stay using the list of hotels provided by the application based on the location fetched by the application and many more. Users with location-aware wireless devices can query about their surroundings (e.g., finding the nearest restaurant or all shopping malls within 5 miles) at any place, anytime. While this ubiquitous computing paradigm brings great convenience for information access, the constraints of mobile environments, the spatial property of location-dependent data, and the mobility of mobile users pose a great challenge for the provision of location-based services to mobile users.

\subsection{Overview}

Online Hotel Parcel and payment system is a mobile application that is dependent on the location of a mobile device, like mobile phone. This application is part of the larger service viz. the location based services (LBS). Virrantaus et al [1] defined LBS services as follows: "Information services accessible with mobile devices through the mobile network and utilizing the ability to make use of the location of the mobile device "Open Geospatial Consortium" [2] defined LBS service similarly: "A wireless-IP service that uses geographic information to serve a mobile user, any application service that exploits the position of a mobile terminal."

A Location Based Service (LBS) is an information and entertainment service, accessible with mobile devices through the mobile network and utilizing the ability to make use of geographical position of the mobile device. A LBS services can be used in a variety of contexts, such as health, work, personal life, etc. LBS include services to identify the location of a person or object, such as discovering the nearest banking cash machine or the where about of a friend or employee. LBS services include parcel tracking and vehicle tracking services. LBS have two major actions, that is:

1. Obtaining the location of user

2. Utilizing this information to provide a service.

These 2 actions are used to answer these 4 questions (below) for a mobile user in a new, fast, and more accurate way, to form the basis for LBS: 
Where am I...?

Where is the nearest ...?

Where is my ...?,

How do 1 get there?

LBS services can be categorized as triggered LBS services (push services) and user-requested LBS services (pull services) [3]. In a triggered (push) LBS service, the location of user's mobile device is retrieved when a condition set in advance is fulfilled. For example, a call to emergency centre can automatically trigger a location request [3]. Advertisement messages can be delivered to users who enter a specific area in a shopping mall, and warning messages can be delivered to users who are in the area where weather conditions will change (e.g. hurricane, rain). In a user-requested (pull) LBS service, the user decides whether and when to retrieve the location of his/her mobile device and use it in the service. User-requested LBS service can involve personal location (i.e. finding the current location of the user) or services location (i.e. finding the location of the nearest restaurant or bank). Navigation and direction system is an example of pull LBS services [3].

\subsection{Components Involved}

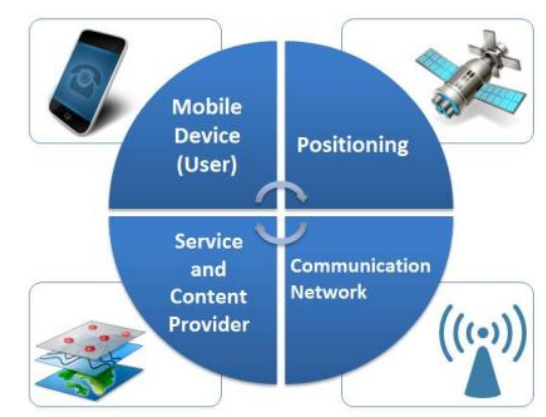

Fig 1.2: Components involved in LBS

Almost all LBSs are based on components present in the Fig 1.2. All of them create LBS infrastructure and parameters of each of them are crucial for the service to work. These components are: Service and Content Provider, Mobile Device, Positioning Systems, and Communication Network.

Service and Content Provider - These are all companies providing service, data and that are responsible for the service request processing. Those providers are for example Location Based Social Networks or GSM operators.

Mobile Device - These are all non-stationary devices that are tools for the user to request the needed information. For example mobile phones, smartphones, PDAs, tablets, laptops. Those devices can have build-in or external positioning module (e.g. GPS antena).

Positioning System - Positioning of the user is of course the heart of LBS. The user position can be obtained in several ways. The most common two of them are GPS with several meters accuracy and the mobile communication network which uses one or more cell towers to determine the position of devices with several hundred meters accuracy. The other possibility is to use the position are WLAN stations - there are companies specialized in capturing information about Wi-Fi location and using it for positioning. This option is possible only in the cities because of need of high network density but it can give surprisingly good accuracy of several dozen meters. Other topic is indoor navigation which can use as well Wi-Fi or Bluetooth for determining position inside buildings.

Communication Network - The last component is communication network which is responsible for transferring the user data and service request from the mobile device to the service provider and then the requested information back to the user.

\subsection{Working Principle}




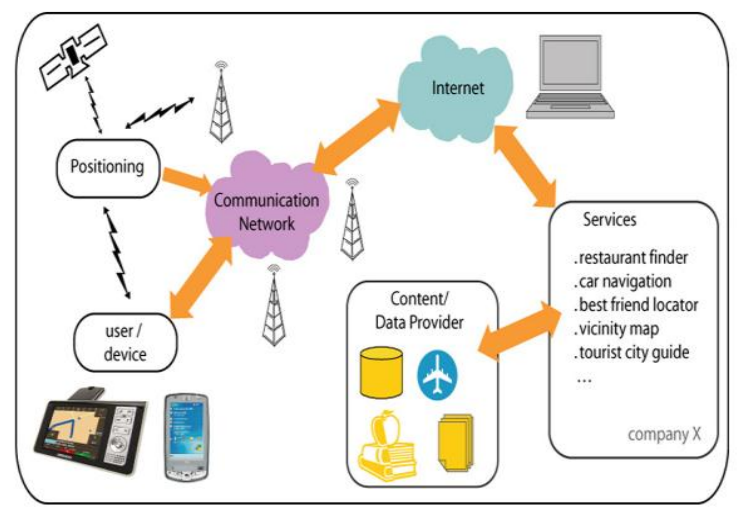

Fig 1.3 - Working Principle of Online Hotel Parcel \& Payment System

The whole product is divided into 4 to 5 functionality. Product supports 3 different types of users namely the Customer, Hotel and System itself. The first basic functionality of the product is to find the exact location of the user when he starts using the application. For this we would be using the mobile GPS and Google Maps to find the users exact location. The extracted location information will be sent to server to find all the listed nearby hotels and their current menus. User will then see the list of all hotels with their menu and users reviews/feedback. User then can explore the hotels and find desired food item which he wants to order as parcel. As part of extra services, system will promote good advertisements to user which will help him to place order quickly. Ones the order is placed, system will inform respective hotel/restaurant to deliver order to the user at the address sent by the user during payment. System will send location of user to hotel so that it will dispatch order to it. System supports PayPal payment gateway to do online payments at the time of paying for the ordered food from the application. For the purpose of transparency the entire security while payment will be handled from the side of PayPal since it's a very trusted online payment source.

\subsection{System Architecture}

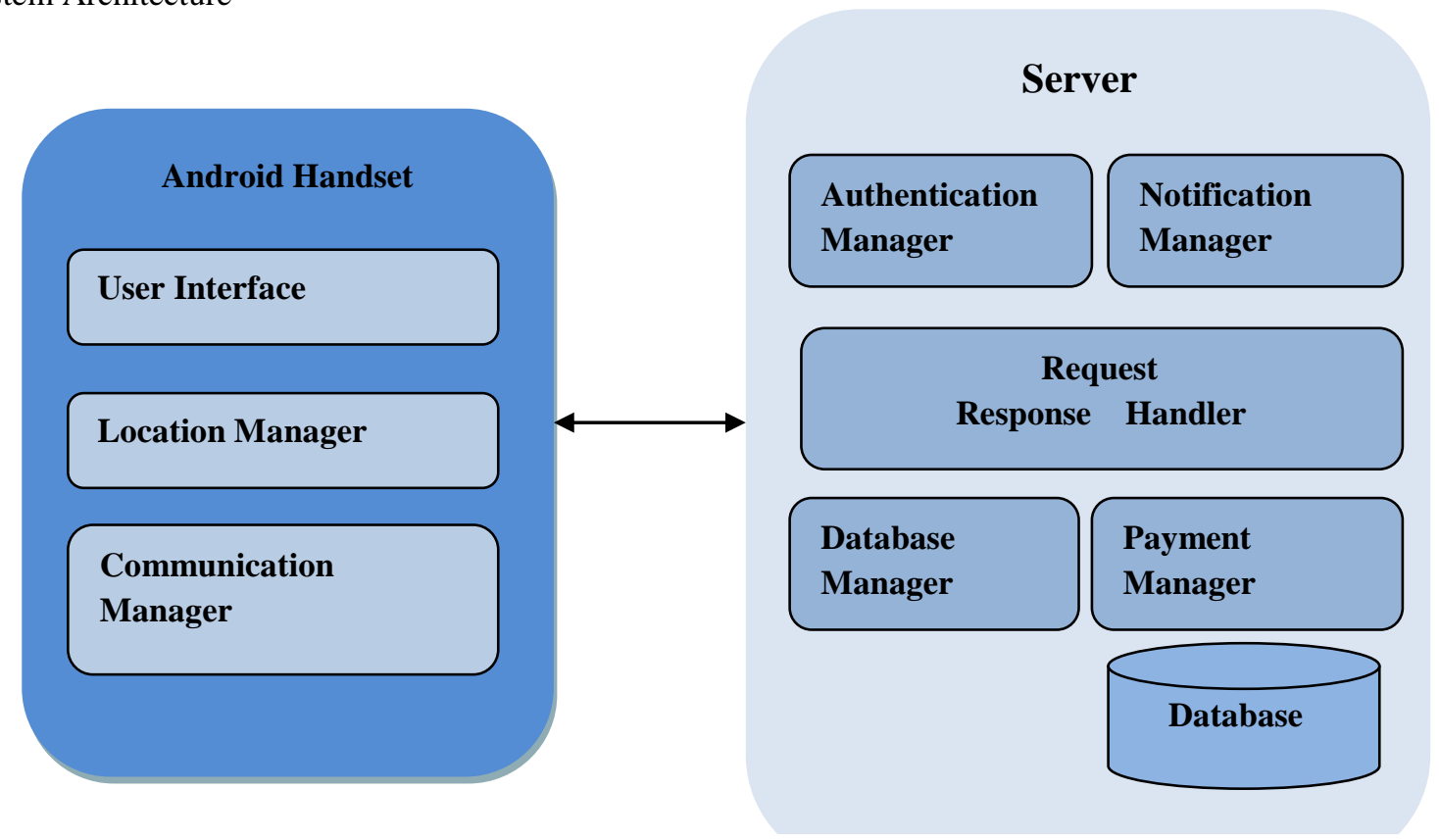

Fig 1.4 - System Architecture showing the communication between the Client (Handset) and the server

The System Architecture depicts the communication between the Android handset and the application server. The Android handset block consists of the User Interface, Google Location Manager and the phones communication manager. The elements involved in the android handset block communicate with the elements of the application server involving the Authentication Manager, Database Manager, Payment Manager, Request 
response handler for handling the user requests and the response from the server and the database itself for storing the user defined locations, previous orders and payments.

The location manager is responsible for the locations provided by the Google Maps such as all the nearby places including restaurants, Cinemas, transport facilities, ATMs and so on. The communication manager is responsible for keeping the communication between the client and the server always online when the application is in use. The authentication manager is responsible for authenticating the user by comparing the credentials provided by the user with the data stored in the database. If the data matches then only the user will be able to use the application. The notification manager keeps the user informed about the status of his order such as whether the order has been dispatched or not. It also notifies that if the placed order is possible at a particular time or not. The request response handler is responsible for handling all the user requests such as placing an order for parcel, retrieving data about nearby places when the location of the user is available to the server and sending it back to the user again. The database manager is responsible for storing the user's transactions such as previous orders, payment history and balance in his PayPal account. The payment manager is responsible for keeping the connection online during a transaction between the client and the PayPal service.

\subsection{Languages and database used}

\subsubsection{Android}

Android consists of a kernel based on the Linux kernel, with middleware, libraries and APIs written in $\mathrm{C}$ and application software running on an application framework which includes Java-compatible libraries based on Apache Harmony. Android uses the Dali virtual machine with just-in-time compilation to run compiled Java code. Android has a large community of developers writing applications ("apps") that extend the functionality of the devices. Developers write primarily in a customized version of Java. There are currently more than approximately 520,000 apps available for Android. Apps can be downloaded from third-party sites or through online stores such as Android Market, the app store run by Google.

One of the most widely used mobile OS these days is ANDROID. Android does a software bunch comprise not only operating system but also middleware and key applications. Android Inc was founded in Palo Alto of California, U.S. by Andy Rubin, Rich miner, Nick sears and Chris White in 2003. Later Android Inc. was acquired by Google in 2005. After original release there have been number of updates in the original version of Android.

Android provides access to the above components to facilitate the implementation of LBS services through the help of following classes;

1. Location Manager

2. Location Provider

3. Geocoding

4. Google-Map

Location Manager - Location Manager Class of android is present to manage all other components needed to establish a LBS system.

Location provider - Location provider represents the technology to determine the physical location i.e. to handle GIS (Geographical Information System). Location Provider component of Android application is a present to facilitate the determination of available provider and selection of suitable one. Finding the List of Available Location Provider, to get a list of names for all the providers available on the device, call get Providers, using a Boolean to indicate if you want all, or only the enabled, providers to be returned:

boolean enabledOnly = true;

List providers = locationManager.getProviders(enabledOnly);

In addition to this GPS provider and Network provider can be accessed directly by using the static variables defined in the LocationManager class:

LocationManager.GPS_PROVIDER

LocationManager.NETWORK_PROVIDER

Furthermore for finding the provider on the basis of some criteria we can use the criteria class and then can find the best provider for defined criteria using the Best Provider Method as shown is the following code snaps:

Criteria criteria $=$ new Criteria () ;

criteria.setAccuracy(Criteria.ACCURACY_COARSE);

criteria.setPowerRequirement(Criteria.POWER_LOW);

// more criteria here

String bestProvider = locationManager.getBestProvider(criteria, true); 
If more than one provider is available fulfilling the given criteria then the one with best performance is returned. On the other hand if no provider is found for the defined criteria then criteria are loosened in order Power use, Accuracy, Ability to return bearing, speed, and altitude.

Geocoding - Reverse geocoding provides a way to convert geographical coordinates (longitude, latitude) into street address and forward geocoding provides a mean to get geographical coordinated from street address. For forward geocoding we use getLatitude() and getLongitude() method as shown is the following code Block; double latitude $=$ location. getLatitude(); double longitude = location .getLongitude () ;

For reverse geocoding we use getFromLocation method with geocoder variable as shown is the following code Block;

//geocod is geocoder variable addresses = geocod.getFromLocation(latitude, longitude, 10);

Google Map in Android - Android provides a number of objects to handle maps in LBS system like MapView which displays the map. To handle this, a MapActivity class is there. To annotate map it provides the overlays class. Even it provides canvas by which one can easily create and display multiple layers over the map.

Moreover, sufficient provisions are there to zoom into the map, localize the map by means of the MapController.

Following code-line shows the Map Handling in Android:

$<$ com.google.android.maps.MapView

android:id="@+id/map_view"

//specify different attributes/>// map controller

MapController mapController $=$ myMapView $\cdot$ getController();

mapController.setCenter(point);

mapController.setZoom(1);

$/ /$ List of present overlays

List $<$ Overlay $>$ overlays $=$ mapView.getOverlays () ;

// adding a new overlays

MyOverlay myOverlay = new MyOverlay () ;

overlays.add(myOverlay);

mapView.postInvalidate();

\subsubsection{SQLlite Database}

SQLlite is an ACID-compliant embedded relational database management system contained in a relatively small C programming library. The source code for SQLlite is in the public domain. Unlike clientserver database management systems, the SQLlite engine is not a standalone process with which the application program communicates. Instead, the SQLlite library is linked in and thus becomes an integral part of the application program. The library can also be called dynamically. The application program uses Slate's functionality through simple function calls, which reduces latency in database access as function calls within a single process are more efficient than inter-process communication. The entire database (definitions, tables, indices, and the data itself) is stored as a single cross-platform file on a host machine. This simple design is achieved by locking the entire database file during writing.

\section{Conclusion}

Initially mobile phones were developed only for voice communication but now days the scenario has changed, voice communication is just one aspect of a mobile phone. There are other aspects which are major focus of interest. Two such major factors are web browser and GPS services. Both of these functionalities are already implemented but are only in the hands of manufacturers not in the hands of users because of proprietary issues, the system does not allow the user to access the mobile hardware directly. But now, after the release of android based open source mobile phone a user can access the hardware directly and design customized native applications to develop Web and GPS enabled services and can program the other hardware components like camera etc. The Online Hotel Parcel \& Payment application will help user to find good restaurants in a short time and the user would be able to order food from the given list of restaurants and the payment will be also made online replacing the usual way of paying with hard cash which in turn would help save time because the entire hotel menu will be at the users addressal and the problem of providing change while accepting delivery of the parcel from the hotel side will not occur. Just like a GPS device its location will also be updated as soon as user changes his/her position and a new list of restaurants would be provided according the new location fetched by the GPS. 


\section{References}

[1] Virrantaus, K., Markkula, J., Garmash, A., Terziyan, V., Veijalainen, J., Katanosov, A., and Tirri, H.Developing GIS supported location-based services. In Web Information Systems Engineering (2001), IEEE, pp. 66_75.

[2] D'Roza, T., and Bilchev, G. An overview of location-based services. BT Technology Journal 21, 1 (2003), 20_27

[3] Schwinger, W., Grin, C., Prll1, B., and Retschitzegger, W. A light-weight framework for location-based services. In Lecture Notes in Computer Science (Berlin, 2005), Springer, pp. 206_210 